\title{
Design and verification of a negative resistance electromagnetic shunt damper for spacecraft micro-vibration
}

\author{
Alessandro Stabile $^{\mathrm{a}}$, Guglielmo S. Aglietti ${ }^{\mathrm{a}}$, Guy Richardson ${ }^{\mathrm{b}}$, Geert Smet ${ }^{\mathrm{c}}$ \\ ${ }^{a}$ Surrey Space Centre, University of Surrey, Guildford, GU2 7XY, UK \\ ${ }^{b}$ Surrey Satellite Technology Ltd. (SSTL), Guildford, GU2 7YE, UK \\ ${ }^{c}$ ESA/ESTEC, Keplerlaan 1, PO Box 299, 2200 AG, Noordwijk, Netherlands
}

\begin{abstract}
Active control techniques are often required to mitigate the micro-vibration environment existing on board spacecraft. However, reliability issues and high power consumption are major drawbacks of active isolation systems that have limited their use for space applications. In the present study, an electromagnetic shunt damper (EMSD) connected to a negative-resistance circuit is designed, modelled and analysed. The negative resistance produces an overall reduction of the circuit resistance that results in an increase of the induced current in the closed circuit and thus the damping performance. This damper can be classified as a semi-active damper since the shunt does not require any control algorithm to operate. Additionally, the proposed EMSD is characterised by low required power, simplified electronics and small device mass, allowing it to be comfortably integrated on a satellite. This work demonstrates, both analytically and experimentally, that this technology is capable of effectively isolating typical satellite micro-vibration sources over the whole temperature range of interest.
\end{abstract}

\section{Introduction}

2

The unprecedented endeavour to build the Hubble Space Telescope in the 3 early 1970's and the desire to achieve the highest-ever imaging resolution bol-

Email address: a.stabile@surrey.ac.uk (Alessandro Stabile) 
stered the research on the modelling and control of micro-vibration on board spacecraft. Micro-vibrations are typically generated by several on-board subsystems and devices, such as reaction wheel assemblies, momentum wheel assemblies, control moment gyros, pointing systems and cryo-coolers. The increasingly stringent stability requirements imposed by advanced, high-resolution payloads have produced an increased interest in the development of betterperforming micro-vibration dampers. Several devices aimed at mitigating microvibrations have been investigated and tested, and depending on the nature of their functioning they can be classified as passive, active or semi-active dampers.

The passive dampers are mostly preferred due to their constructive simplicity, relative low cost and reliability. Among this kind of damper, viscoelastic materials are the cheapest and lightest damping solutions and are widely used by companies in the space sector $[1,2,3]$. However, the limited loss factors achievable with these materials, along with the strong dependency on the operating temperature and frequency ranges, make the design of viscoelastic dampers quite complex. Other passive dampers studied in the last 25 years include: Eddy-current dampers [4, 5, 6], D-struts [7, 8] and piezoelectric transducers shunted with passive electrical networks $[9,10]$. Nevertheless, it is still a challenge to employ pure passive isolation techniques to dampen low frequency micro-vibrations onboard a spacecraft [11].

Active control techniques [12] and active dampers are sometimes necessary in order to achieve more stringent conditions of isolation. Active dampers are fully controllable and capable of producing higher-order vibration attenuation with respect to passive dampers, but they also require a significant amount of power to operate. Active isolation can be obtained by using external actuators and sensors to provide control forces and feedback signals. Piezoelectric patches have been used extensively in active vibration control applications $[13,14,15]$, and thanks to the development of the self-sensing technique these smart materials can be employed simultaneously as sensors and actuators $[16,17]$. The main advantages of this technique are the realization of a very robust, trulycollocated control (given by the sensor and the actuator being placed in the 
same position) as well as the reduction of system mass and complexity due to the elimination of independent sensors. However, variations of the piezoelectric properties produced by environmental and operating conditions would require a continual tuning of the circuit that, if not effectuated, could destabilise the closed-loop system. Also, electromagnetic transducers can be used either as velocity sensors or force actuators $[18,19]$, or simultaneously as both in the selfsensing technique [20, 21]. These transducers are characterised by large strokes, high bandwidth and low cost, but they may also suffer serious spillover (e.g. actuator saturation) and stability issues.

Recently, the use of negative impedances was proved to considerably improve the damping performance when connected to piezoelectric transducers $[22,23]$ or electromagnetic transducers $[24,25,26]$. Negative impedances are used to cancel or reduce the inherent electrical properties of the transducers (e.g. piezoelectric patches are characterised by an inherent capacitance). In the case of electromagnetic transducers (characterised by an inductance and a resistance), the reduction of the overall resistance by means of the negative resistance increases the induced current flowing in the circuit and thus the damping performance.

This paper presents an electromagnetic shunt damper (EMSD) connected to a negative-resistance circuit, and demonstrates its vibration attenuation performance by applying the isolator to a single-degree-of-freedom (SDOF) system. Although the shunt circuit requires power to operate, this type of EMSD can be considered as a semi-active damper for two reasons: i) the negative-resistance circuit acts overall as a passive electrical component having a constant negative magnitude, without requiring any control algorithm; ii) the damper requires little external power to function because part of the energy is self-provided by the relative motion between the magnet and the coil (i.e. the damper would work as a passive damper if the coil terminals were short-circuited). The proposed damper is analysed not only in terms of damping performance, but also as a satellite subsystem that needs to withstand certain physical and environmental criteria. Therefore, the mass and power budgets of the damper have been 
taken into account, as well as stability conditions derived from considering an operating temperature range from $-20{ }^{\circ} \mathrm{C}$ to $+50{ }^{\circ} \mathrm{C}$.

This work differs from previous articles on similar dampers [24, 26, 27] by proving, both analytically and experimentally, that this technology is able to effectively isolate typical sources of micro-vibration in space (in the region of $5 \mathrm{~kg}$ ) without using any active control algorithm to adjust for changes in the environmental conditions. In fact, the damper transmissibility shows a final decay rate of $-40 \mathrm{~dB} / \mathrm{dec}$, while drastically reducing the force amplification at the resonance frequency throughout the whole temperature range of interest. Therefore, the improved damping performance, along with the low power required to operate it and an overall mass that is less than $4 \%$ of the suspended mass, are among the main features of the proposed EMSD, which make it highly competitive when compared with other well-established micro-vibration dampers.

The rest of the paper is organised as follows. Section 2 presents the analytical model of the proposed EMSD. Section 3 describes the experimental set up. Section 4 reports the tests results and shows the correlation with the analytical data. The conclusions are drawn in Section 5.

\section{Analytical model}

A schematic of the system studied in this paper is shown in figure 1 . This model consists of a mass suspended on a metal spring with stiffness coefficient $k$ and an EMSD. The damper consists of two electromagnets connected together and rigidly attached to a fixed boundary, a cylindrical magnetic assembly bonded to the suspended mass and coaxially aligned with the electromagnets, and a shunt impedance connected to the terminals of the coils. The closed electric circuit formed by the electromagnets and the shunt can be equivalently represented as a voltage source $V_{0}$ in series with an inductance $L_{e}$ and two resistances represented by the resistance of the electromagnet, $R_{e}$, and the equivalent resistance of the shunt, $R_{s}$. It is noted that a SDOF system is not representative of the actual behaviour of a reaction wheel, which produces disturbances in all 
six degrees of freedom. However, this configuration is aimed at obtaining a first assessment of the damper performance that can then be compared with other well-established damping solutions $[3,18,28]$. In particular, special attention is given to the transfer function between the input force (i.e. the disturbance forces produced by the reaction wheels) and the force transmitted to the ground. In this study, the mass of the satellite where the reaction wheels are connected to has been assumed to be several orders of magnitude bigger than the reaction wheels, and hence the ground can be considered fixed in space.

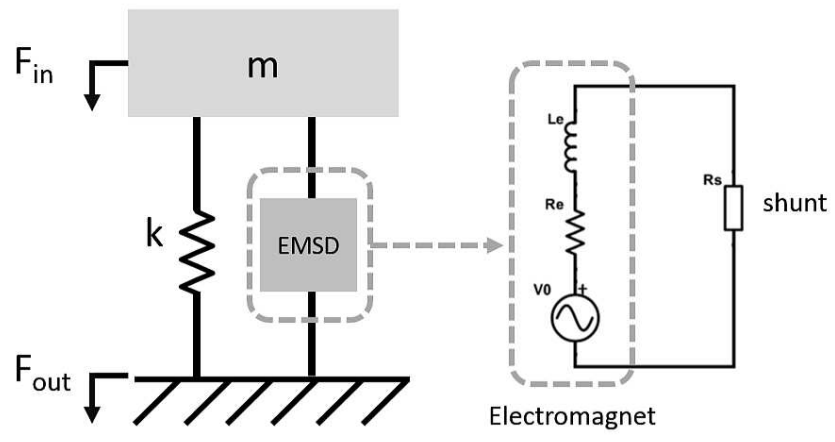

Figure 1: Schematic representation of the SDOF model, with the electrical schematic of the shunt circuit of the EMSD

\subsection{State-space modelling}

EMSDs function through the combination of two different physics phenomena that are described by the Faraday-Lenz law and the Lorentz force law. The Faraday-Lenz law asserts that a relative velocity between an electromagnet and a permanent magnet induces an electromotive force $V_{0}$ (i.e. electric voltage) at the terminals of the electromagnet. This induced voltage can be expressed with the equation:

$$
V_{0}=\oint(\vec{v} \times \vec{B}) \cdot d \vec{l}
$$


where $\vec{v}$ is the magnet velocity with respect to the electromagnet, $\vec{B}$ is the magnetic field and $d \vec{l}$ is the infinitesimal length of the coil turns along which the integral is computed. Considering a cylindrical coordinate system $(\hat{r}, \hat{\phi}, \hat{z}$, with the z-axis along the magnet's longitudinal axis), and the only relative motion between the conductor and the magnet to be along the z-axis, the velocity, $v$, and the magnetic field, $B$, can be written as:

$$
\begin{aligned}
& \vec{v}=0 \hat{r}+0 \hat{\phi}+v_{z} \hat{z} \\
& \vec{B}=B_{r} \hat{r}+B_{\phi} \hat{\phi}+B_{z} \hat{z}
\end{aligned}
$$

This results in an electromotive force equal to:

$$
V_{0}=\oint\left(v_{z} B_{r} \hat{\phi}-v_{z} B_{\phi} \hat{r}\right) \cdot r d \vec{\phi}=\oint v_{z} B_{r} r d \phi
$$

where it can be seen that the magnetic field contributes to the induced voltage only with the radial component.

In the micro-vibration load case, it can be assumed that the magnetic field seen by the conductive material is constant in time, since the relative displacement is in the order of magnitude of tenths of a millimetre, and so its variations are almost negligible. Also, given the small cross section of the electromagnet, one can assume a linear trend of the magnetic field along the radial axis inside the coil. Therefore, Eq.(3) can be simplified as:

$$
V_{0}=2 \pi n_{t} r_{a v g} \bar{B}_{r} v_{z}=K_{d} v_{z}
$$

where $n_{t}$ is the number of turns of the coil, $r_{\text {avg }}$ is the average radius of the conductor, and $\overline{B_{r}}$ is the average radial component of the magnetic field through the coil cross section. $K_{d}$ is defined as the electro-mechanical transducer coefficient.

Once the induced current is generated, it couples with the surrounding magnetic field to produce the Lorentz force. This force is described by the equation:

$$
\vec{F}_{d}=\oint I d \vec{l} \times \vec{B}
$$

Adopting the same assumptions used for the electromotive force, and since $d \vec{l}$ has the only component along $\hat{\phi}$, the damping force $F_{d}$ can be expressed as:

$$
\vec{F}_{d}=-2 \pi n_{t} r_{a v g} \bar{B}_{r} I \hat{z}=-K_{d} I \hat{z}
$$


These two laws considered together demonstrate that the force produced by a permanent magnet which moves close to a conductive material is proportional in magnitude and opposite in direction to their relative velocity. Therefore, this kind of force opposes the movement of the magnet and acts like a damping force.

The system shown in Fig. 1 can be modelled by including Eqs.(4) and (6) in a fully-coupled system of four equations:

$$
\left\{\begin{array}{l}
m \ddot{z}+k z=F_{d}+F_{i n} \\
V_{0}=K_{d} v_{z} \\
L_{e} \dot{I}+\left(R_{e}+R_{s}\right) I=V_{0} \\
F_{d}=-K_{d} I
\end{array}\right.
$$

which respectively represent: the equation of motion (the input force is described by the term $F_{i n}$ ), the Faraday-Lenz law of induction, Kirchhoff's voltage law associated with the electric circuit, and the Lorentz force.

Through the assumptions of micro-vibration and steady-state conditions, the system shown in Eq.(7) can be linearised and rearranged via a state-space representation. The use of state space modelling considerably simplifies the analysis of the system in the frequency domain into which it can be converted by taking the Laplace transform. The state-space model can be written as

$$
\begin{gathered}
{\left[\begin{array}{c}
\dot{z} \\
\ddot{z} \\
\dot{I}
\end{array}\right]=\left[\begin{array}{ccc}
0 & 1 & 0 \\
-k / m & 0 & -K_{d} / m \\
0 & K_{d} / L & -\left(R_{e}+R_{s}\right) / L
\end{array}\right]\left[\begin{array}{l}
z \\
\dot{z} \\
I
\end{array}\right]+\left[\begin{array}{c}
0 \\
1 / m \\
0
\end{array}\right] F_{\text {in }}} \\
Y=\left[\begin{array}{lll}
k & 0 & K_{d}
\end{array}\right]\left[\begin{array}{l}
z \\
\dot{z} \\
I
\end{array}\right]+[0] F_{\text {in }}
\end{gathered}
$$

where the state vector consists of three state variables: mass displacement $(z)$, mass velocity $\left(\dot{z}=v_{z}\right)$, and circuit current $(I)$. The output vector $Y$ represents the force transmitted to the satellite structure. This single-input-single-output (SISO) system can be converted to the frequency domain, and solved for the 
force transfer function (TF). The TF considered in this case is the relation between the input force $F_{i n}$ and the force transmitted to the ground. The TF is characterised by poles and zeros that describe the system's dynamic behaviour in the frequency domain. In the case of a mass-spring system, the TF is only characterised by a couple of complex-conjugate poles, which determine the resonance peak and the roll-off slopes of $-40 \mathrm{~dB} / \mathrm{dec}$. Adding to this system a magnetic damper with almost negligible inductance (e.g an Eddy-current damper as shown in Ref. [29]) produces a reduction of the resonance peak but also the introduction of a real zero that brings the decay rate to $-20 \mathrm{~dB} / \mathrm{dec}$. The EMSD proposed in this paper presents a relatively high resistance-overinductance ratio that introduces a real pole at low frequency, thus causing the roll-off slope to be $-40 \mathrm{~dB} / \mathrm{dec}$, while having a considerable reduction of the resonance peak.

\subsection{Magnet configuration}

The damper performance can be improved, besides other means, through the increasing of the electro-mechanical transducer coefficient, $K_{d}$. As shown in Eq.(4), $K_{d}$ depends on the radial component of the magnetic field. A trade off between various configurations of the magnetic assembly in order to increase $\bar{B}_{r}$ was conducted through the software COMSOL Multiphysics. Three different configurations were analysed: a single cylindrical magnet (see Fig. 2a), a single cylindrical magnet with two iron yokes at the ends (see Fig. 2b) and three cylindrical magnets arranged with opposing polarity and alternated with two iron yokes (see Fig. 2c). The last configuration uses a non-magnetic, M4 screw to keep the magnets in place. The cross-section of the two electromagnets was kept constant throughout the analysis, and the diameter of the coils was changed in order to maintain a safe gap of $1 \mathrm{~mm}$ between the magnet and the coils. A parameter defined as the ratio of the coefficient $K_{d}$ to the mass of the magnetic assembly was used to compare these configurations. Tab. 1 shows the main parameters for each configuration. The magnetic assembly shown in Fig. 2c and $2 \mathrm{f}$ was ultimately chosen not only for the better $K_{d}$-over-mass parameter, but 


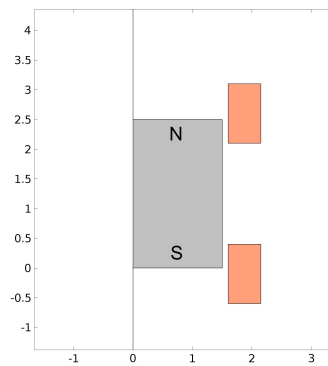

(a)

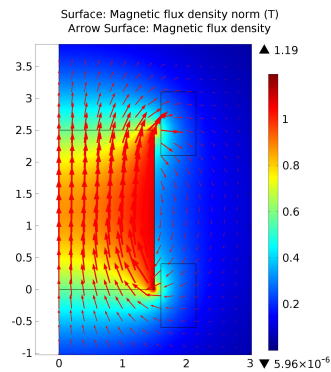

(d)

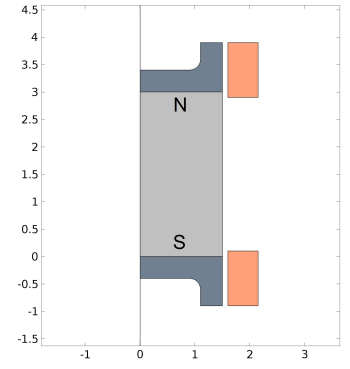

(b)

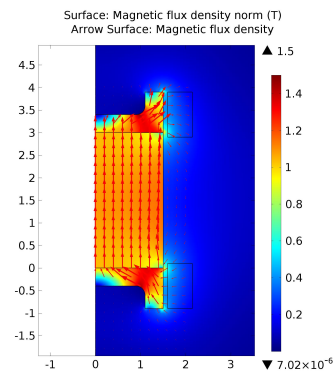

(e)

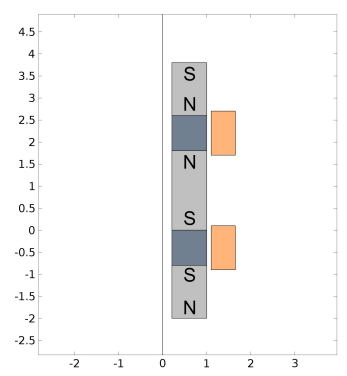

(c)

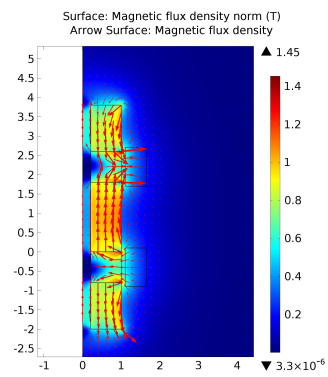

(f)

Figure 2: Comparison between three different configurations of the magnetic assembly. The analyses were carried out through COMSOL Multiphysics using a 2D-axisymmetric formulation. The top row shows the disposition of the magnets and yokes, and the magnetic polarization, whereas the bottom row shows the magnetic flux density. (a,d) single cylindrical magnet; (b,e) single cylindrical magnet with two iron yokes at the ends; (c,f) three cylindrical magnets arranged with opposing polarity and alternated with two iron yokes.

\subsection{Negative resistance}

The use of negative resistance in the shunt $\left(R_{s}\right)$ produces a reduction of the overall circuit resistance that results in an increase of the electric current, $I$, and hence of the damping force, $F_{d}$. Several examples of the use of negative impedances have been found in literature [26, 27], but these circuits are always implemented using a controller board (e.g. dSpace R1103) connected to an elaborated circuitry to obtain the desired value of the impedance (the electronic 


\begin{tabular}{lccc}
\hline Property & \multicolumn{3}{c}{ Value } \\
\hline & Config. 1 & Config. 2 & Config. 3 \\
\hline $\bar{B}_{r}(\mathrm{~T})$ & 0.20 & 0.23 & 0.28 \\
$K_{d}(\mathrm{~N} / \mathrm{A})$ & 6.45 & 7.41 & 6.7 \\
$K_{d} /$ mass $\left(\frac{N / A}{K g}\right)$ & 47.78 & 32.21 & 49.63 \\
\hline
\end{tabular}

Table 1: trade-off of magnetic assembly

board from Ref. [26] is reported in Fig. 3). Moreover the evaluation of the power required by the shunt has never been taken into account. In this study, the negative resistance is created by utilising an analogue circuit called negative impedance converter [30]. It consists of a single operational amplifier (op-amp) and three resistors that are connected as shown in Fig. 4. The equivalent resistance of the shunt is,

$$
R_{s}=-R_{1}\left(\frac{R_{3}}{R_{2}}\right)
$$

By considering the resistances $R_{2}$ and $R_{3}$ to be equal to each other, the shunt produces a negative resistance $R_{s}=-R_{1}$. In terms of stability, this circuit tends to be stable as long as the resistance of the electromagnet, $R_{e}$, is greater in magnitude than the shunt resistance, $R_{1}$ (this aspect will be taken into account in the next paragraph). The supplied power required by the shunt circuit can be expressed as

$$
P=\left(V^{+}-V^{-}\right) \cdot\left(I_{q}+I_{o}\right)
$$

where $V^{+}-V^{-}$is the total supply voltage, $I_{q}$ is the quiescent current consumed by the op-amp, and $I_{o}$ is the op-amp output current. $I_{o}$ can be approximated to the current flowing in the resistor $R_{1}$, because the choice of selecting $R_{2}$ and $R_{3}$ to be three orders of magnitude greater than $R_{1}$ produces a current, $I_{R_{2} R_{3}}$, that can be neglected. Since the signal is sinusoidal, the rms value of the power can be obtained as: 


$$
P_{a v g}=\left(V^{+}-V^{-}\right) \cdot\left(I_{q}+I_{r m s}\right)
$$

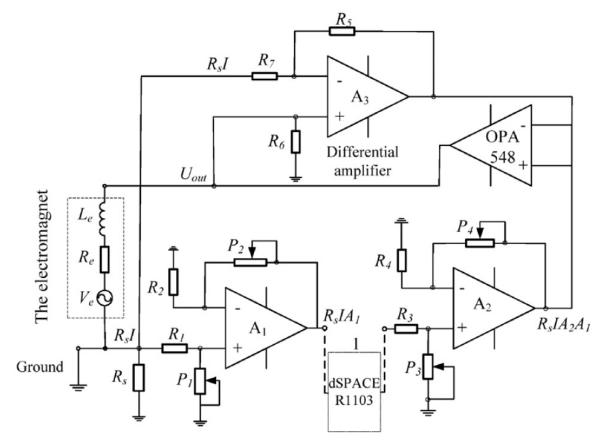

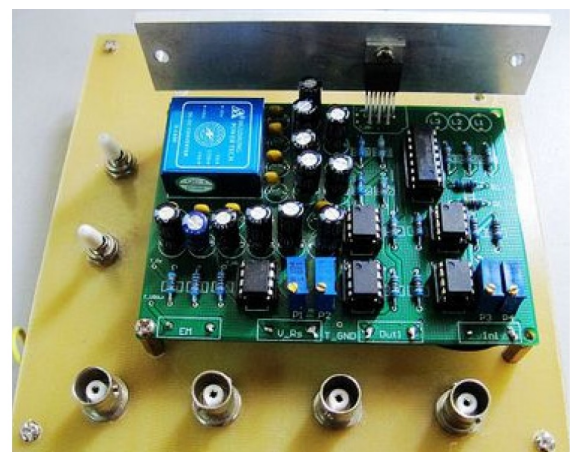

Figure 3: Block diagram and circuit board of the negative resistance shunted impedance presented in Ref. [26]

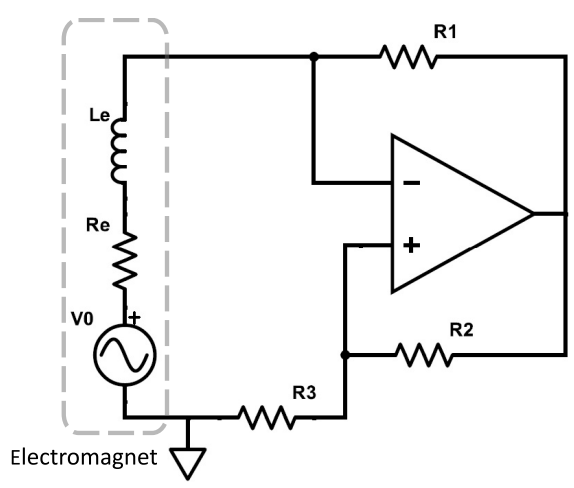

Figure 4: Electrical schematic of the negative resistance impedance converter connected to the electromagnet 


\subsection{Parametric trade-off and expected results}

The environmental conditions at which a satellite operates can usually affect the performance of dampers (e.g. viscoelastic materials are strongly dependent on the surrounding temperature). The suspended mass has been chosen to be 5 $\mathrm{kg}$ and corresponds approximately to the mass of two 100SP-O reaction wheels used by Surrey Satellite Technology Ltd (SSTL). The operating temperature range for this type of reaction wheel is from $-20^{\circ} \mathrm{C}$ to $+50^{\circ} \mathrm{C}$ [1]. Therefore, there is an increasing interest in the development of damping solutions that guarantee good isolation performances in this temperature range. An EMSD is made uniquely of metal parts (Nd-Fe-B magnets and copper) whose characteristics with respect to temperature variations are well established. In particular, the resistivity of the copper increases linearly with respect to the temperature with a thermal coefficient of $0.00386{ }^{\circ} \mathrm{C}^{-1}$. Also, the residual induction of the $\mathrm{Nd}-\mathrm{Fe}-\mathrm{B}$ magnet is characterised by a linear temperature coefficient of -0.0012 ${ }^{\circ} \mathrm{C}^{-1}$. These two effects have been taken into account for the assessment of the damper performance.

Regarding the negative resistance circuit, the three resistors $R_{1}, R_{2}$ and $R_{3}$ can be chosen among space-qualified, off-the-shelf components that have tolerances down to $0.005 \%$ and temperature coefficients of $1 \cdot 10^{-6}{ }^{\circ} \mathrm{C}^{-1}$. Hence these resistors can be considered constant over a wide temperature range when compared with the electromagnet resistance, $R_{e}$. As already stated above, the EMSD would become unstable if the total resistance $\left(R_{e}+R_{s}\right)$ is negative, and this could happen at the lowest temperature range limit (where $R_{e}$ reaches its minimum value). In order to prevent this, a minimum value of $0.1 \Omega$ was imposed on the total resistance at a temperature of $-20{ }^{\circ} \mathrm{C}$. A trade-off of the damper parameters was performed to meet the stability requirement and also to guarantee an amplification at the resonance frequency to not be greater than $6 \mathrm{~dB}$ throughout the whole temperature range. The corner frequency (i.e. the point in the TF where the output is half the value $(-3 \mathrm{~dB})$ of the input) was set to be equal to $10 \mathrm{~Hz}$ for this test case. The trade off was carried out by analysing the system at three different temperatures: $-20{ }^{\circ} \mathrm{C},+50{ }^{\circ} \mathrm{C}$ and the 
range mid point, $+15^{\circ} \mathrm{C}$.

During the analysis it was assumed that the energy dissipated by the coil through Joule heating was removed from it (only through conduction due to the absence of atmosphere in space) so that the electromagnet did not change its temperature with respect to the surrounding environment. This assumption is corroborated by the fact that only a small amount of thermal power needs to be dissipated in a typical micro-vibration load case (see Section 3). Also, this hypothesis allows to study the system in steady-state conditions using Eq.(8), where the magnitudes of the coil resistance, $R_{e}$, and the average radial component of the magnetic field, $\bar{B}_{r}$, are determined by the initial environmental temperature and are constant throughout the analysis. The magnitudes of $R_{e}$ and $\bar{B}_{r}$ at $+15^{\circ} \mathrm{C}$ were taken as reference values, and a thermal analysis was performed in COMSOL Multiphysics to evaluate their variations within the temperature range of interest. The final choice of the parameter set that has met the predefined requirements is reported in Tab. 2.

\begin{tabular}{|c|c|c|c|c|}
\hline Property & & \multicolumn{3}{|c|}{ Value } \\
\hline Mass, m (kg) & & \multicolumn{3}{|c|}{5} \\
\hline Spring Stiffness, k (N/m) & & \multicolumn{3}{|c|}{4600} \\
\hline Coil Inductance, $\mathrm{L}_{e}(\mathrm{mH})$ & & \multicolumn{3}{|c|}{1.3} \\
\hline Shunt Resistance, $\mathrm{R}_{s}(\Omega)$ & & \multicolumn{3}{|c|}{-1.5} \\
\hline & Temp. & $-20{ }^{\circ} \mathrm{C}$ & $15{ }^{\circ} \mathrm{C}$ & $50{ }^{\circ} \mathrm{C}$ \\
\hline Coil Resistance, $\mathrm{R}_{e}(\Omega)$ & & 1.60 & 1.85 & 2.10 \\
\hline E-M Transducer Coefficient, $\mathrm{K}_{d}$ (N/A) & & 6.98 & 6.7 & 6.42 \\
\hline
\end{tabular}

Table 2: Final choice of the parameter set obtained through a trade-off

A comparison of the force TFs between the system without damping and 
the system with the EMSD is shown in Fig. 5. It can be seen that the use of the EMSD produces the expected reduction of the resonance peak, and presents an amplification that is always below $6 \mathrm{~dB}$ in all three cases studied. The corner frequency is equal to $10 \mathrm{~Hz}$ for the case at $15{ }^{\circ} \mathrm{C}$, but it moves slightly within the temperature range, going from $8 \mathrm{~Hz}$ to $20 \mathrm{~Hz}$. However, at high frequency the three curves almost overlap, showing the final slope of $-40 \mathrm{~dB} / \mathrm{dec}$ that is typical of a mass-spring system.

The shift of the corner frequency at low temperatures is due to the swap of the complex-conjugate poles with the real pole introduced with the proposed damper. As stated in Section 2.1, reducing the overall resistance produces an increase of the damping ratio associated with the complex-conjugate poles and moves the real pole toward lower frequencies. However, when the overall resistance decreases beyond a certain value and tends to zero the electric circuit becomes less effective in dissipating energy through Joule heating, which corresponds to the reduction of the damping performance. Also, the increased current produces a stronger magnetic field with opposite polarity with respect to the permanent magnet. The repulsive force produced by these two opposite magnetic fields is directly proportional to the displacement (i.e. it behaves like a spring [31]), which results in the increase of the system stiffness. Therefore, reducing beyond a certain value the overall resistance produces eventually the reduction of the damping force and the appearance of the spring-like behaviour. In the system under examination, the resistance of $0.1 \Omega$ reached at the temperature of $-20^{\circ} \mathrm{C}$ causes the swap of the poles. However, this phenomenon does not compromise the EMSD damping performance since the maximum amplification is kept below $6 \mathrm{~dB}$.

\section{Experimental set-up}

\subsection{Mechanical test rig}

A laboratory test rig was built in house. It was designed to satisfy the requirement of the mass having only a single DoF, while trying to avoid the 


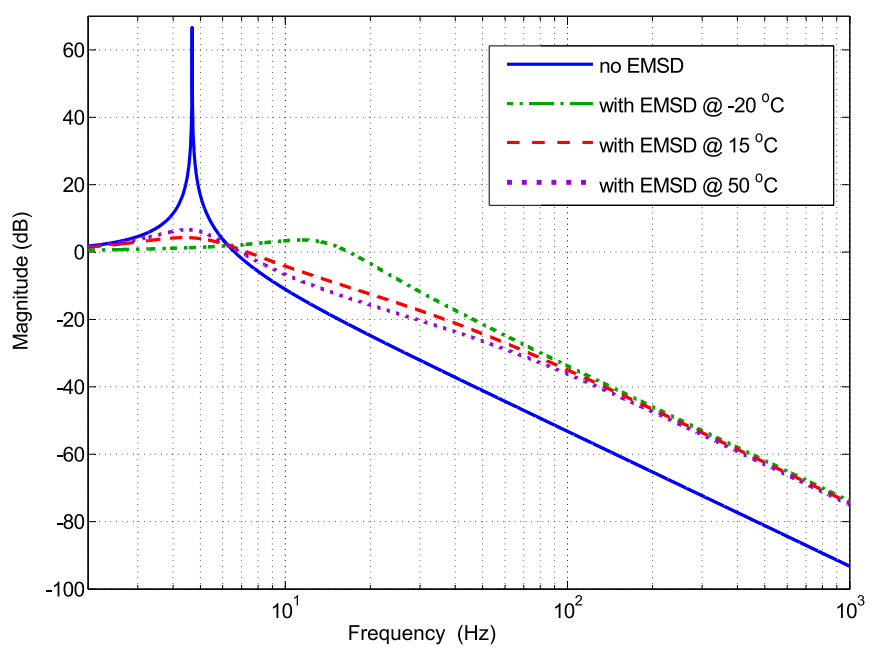

Figure 5: Comparison of the force TFs between the system without EMSD and the system with EMSD at three different temperatures

test rig's vibration modes interfering with the data acquisition. The mass of 5 $\mathrm{kg}$ was obtained through the combination of a dummy mass formed by three steel plates bolted together $(4.85 \mathrm{~kg})$ and the mass of the magnetic assembly $(150 \mathrm{~g})$. It is noted that the magnetic assembly and the two electromagnets weighted $190 \mathrm{~g}$ overall, which represents less than $4 \%$ of the total suspended mass. In order to simulate microgravity, the dummy mass was offloaded using bungee cords characterised by a sub-hertz resonance frequency. A suspended mini-shaker has then been connected to the centre of the dummy mass so that the vertical disturbance of a reaction wheel was reproduced. Steel flexures were used to guarantee that the mass could only move in the vertical direction, whereas displacements/rotations along the other five DoFs were minimised. The fully-assembled test rig bolted on a Kistler table is shown in Fig 6. A model of the test rig was built on Patran and analysed with Nastran (see Fig. 7). This finite element analysis showed that the test rig behaves as a SDOF up to $150 \mathrm{~Hz}$, where secondary modes in the flexures are excited, affecting the force TF. Ideally, these secondary modes were desired to be above $300 \mathrm{~Hz}$, but the 
dimensions of the Kistler table constrained the design of the flexures.

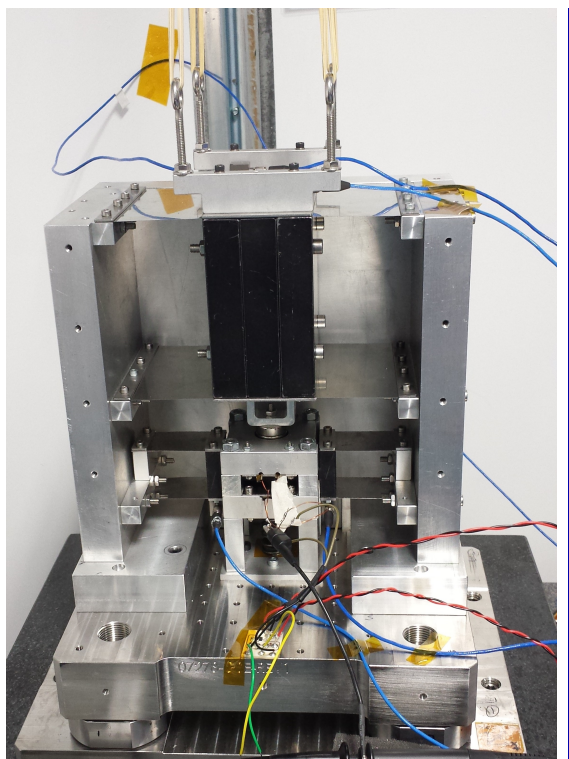

(a)

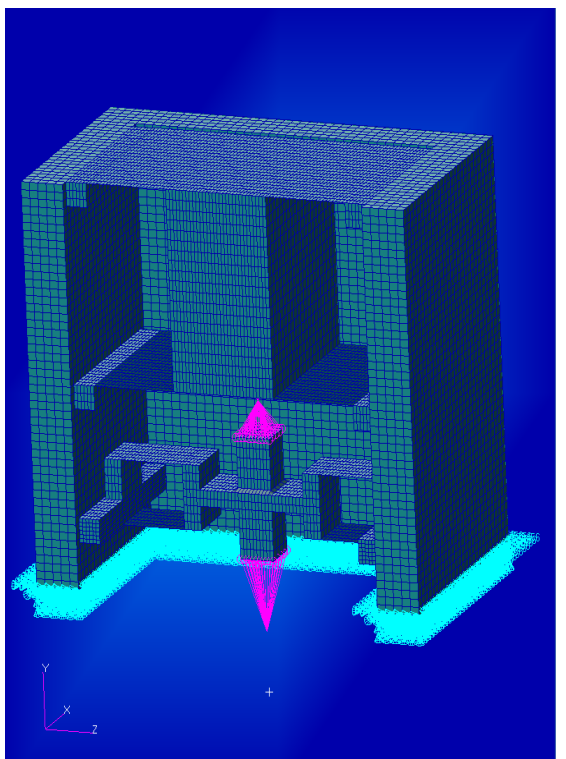

(b)

Figure 6: Experimental test rig. (a) test rig mounted on the Kistler table; (b) Patran model of the test rig

\subsection{EMSD manufacturing}

The design and realisation of the EMSD required an appropriate selection of the circuit components in order to reproduce the same conditions simulated in the analytical model. The clearance of $1 \mathrm{~mm}$ between the magnet and the coil (as already shown in Section 2.2) was chosen as safety margin to prevent the two elements to get in contact in case the device will be tested for the launch vibration environment. The choice of using self-bonding magnet wire allowed to fulfill the 1-mm-gap requirement. This type of wire is coated with an additional layer of adhesive polymer that is activated by heat or solvents. Once activated the adhesive bonds adjacent turns of wire together, forming a compact selfsupporting coil. In this way, the inner spindle can be removed, and any extra spacing between the magnet and the coil is eliminated. 


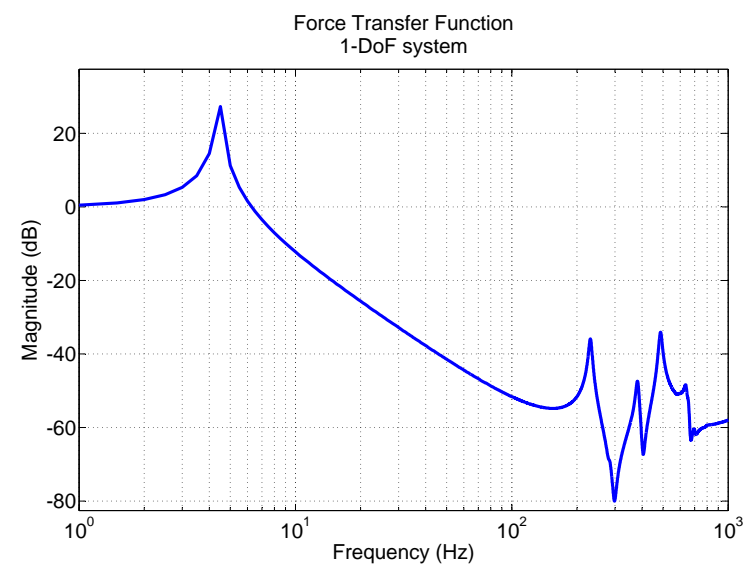

Figure 7: Force transfer function of the system without EMSD obtained through FEM (Nastran).

Another fundamental step was the realization of the negative-resistance electric circuit. Based on the analysis made with the freely-available LTSpice program, several op-amps have been investigated to verify that they performed as expected. Some of the required features include low quiescent current (which drives the minimum supplied power for the op-amp to operate), low input noise and the output current to be at least $50 \mathrm{~mA}$. The LT1722 op-amp was finally selected as it met all the desired requirements (see Tab. 3).

\section{Experimental results and discussions}

The present study verifies the analytical model of Eq.(7) with experiments. The test rig was mounted on a Kistler table. The Kistler table isolation system (a suspension system lays underneath the table acting as a low pass filter) presented an $8-\mathrm{Hz}$ resonance mode that required the tests to be divided into two different sine sweeps: a low-frequency one without the suspension system engaged, and a high-frequency one with the isolation system. The mini-shaker was controlled through a force feedback loop to generate first a 0.5 -N-amplitude sinusoidal input force sweeping from $2 \mathrm{~Hz}$ to $20 \mathrm{~Hz}$, and then a 1-N-amplitude 


\begin{tabular}{lcc}
\hline Property & Typ & Max \\
\hline Input Noise Voltage $(n V / \sqrt{H z})$ & 3.8 & \\
Quiescent Current $(\mathrm{mA})$ & 3.7 & \\
Output Current $(\mathrm{mA})$ & 50 & $80^{\mathrm{a}}$ \\
Supply Voltage $(\mathrm{V})$ & \pm 5 & \pm 6.3 \\
\hline
\end{tabular}

${ }^{a}$ Value measured experimentally.

Table 3: Main features of the LT1722 op-amp

sinusoidal input force sweeping from $15 \mathrm{~Hz}$ to $350 \mathrm{~Hz}$. The input force of the first sine sweep was set to be $0.5 \mathrm{~N}$ so as to prevent the flexures from operating in the nonlinear region due to relatively large displacements (e.g. in the order of millimetres). The lab temperature was approximately $18{ }^{\circ} \mathrm{C}$. The comparison between the experimental results and the analytical data (after tweaking the parameter set accordingly to the lab temperature) is shown in Fig. 8. Through the observation of the test results for the system with the shunt circuit disconnected (see Fig. 8a), it has been able to asses the test rig's mechanical damping that was initially omitted from the analytical model. This damping naturally occurs in a mechanical assembly and can be produced by several factors (e.g. micro-friction between adjacent components, air resistance, hysteresis damping in the steel flexures and Eddy-current damping due to the relative motion between the magnet and other surrounding conductive materials, like aluminium). By adding a small amount of viscous damping in Eq. (7) (about $6 \%$ of the critical damping), it can been observed that good correlation between the analytical data and the experiments has been achieved. The behaviour of the system above $180 \mathrm{~Hz}$ is characterised by some resonance peaks that, as expected through the Nastran analysis, are attributable to the secondary vibration modes of the flexures. Good correlation can also be observed for the system with the EMSD (see 
Fig. 8b). As already shown in Fig. 5, the TF shows a maximum amplification of $4.12 \mathrm{~dB}$, a cut-off frequency at about $10 \mathrm{~Hz}$, and a roll-off slope of $-40 \mathrm{~dB} / \mathrm{dec}$. For this case, it can be seen that the additional mechanical damping that was previously added has almost a negligible effect on the damper performance.

The analytical model was further evaluated through the analysis of two other TFs. The relations between the input force and the mass velocity (for the case without the EMSD) and the induced current (for the system with the EMSD) can be obtained by modifying the output vector in Eq. (9) as follows:

$$
Y=\left[\begin{array}{l}
Y_{v} \\
Y_{c}
\end{array}\right]=\left[\begin{array}{lll}
0 & 1 & 0 \\
0 & 0 & 1
\end{array}\right]\left[\begin{array}{l}
z \\
\dot{z} \\
I
\end{array}\right]+\left[\begin{array}{l}
0 \\
0
\end{array}\right] F_{i n}
$$

These two outputs are measured respectively through accelerometers placed on top of the dummy mass and an oscilloscope probe connected to the coil's ends. The comparison of these analytically-obtained TFs with the experimental data is shown respectively in Figs. 9 and 10. The good correlation that can be observed for both TFs, along with the use of different kinds of sensors, are further confirmations of the accuracy of the analytical model and also of the correct functioning of the shunt circuit.

The maximum power required by the shunt $(0.53 \mathrm{~W})$ was registered at 5.60 $\mathrm{Hz}$, where the highest current was drawn from the power supply. In particular, the current flowing in the electromagnets had an amplitude of $70 \mathrm{~mA}$, and using Eq. (12) the average power required by the shunt at $5.60 \mathrm{~Hz}$ is obtained as:

$$
P_{a v g}=\left(V^{+}-V^{-}\right) \cdot\left(I_{q}+I_{r m s}\right)=10 \mathrm{~V} \cdot(3.8 m A+0.707 \cdot 70 m A)=0.53 \mathrm{~W}
$$

This value of the average power of this semi-active system is considerably lower when compared with active isolation methods, where actuators and sensors need to be driven (e.g. in Ref. [14] the single strut requires a minimum of 15 W to operate). 


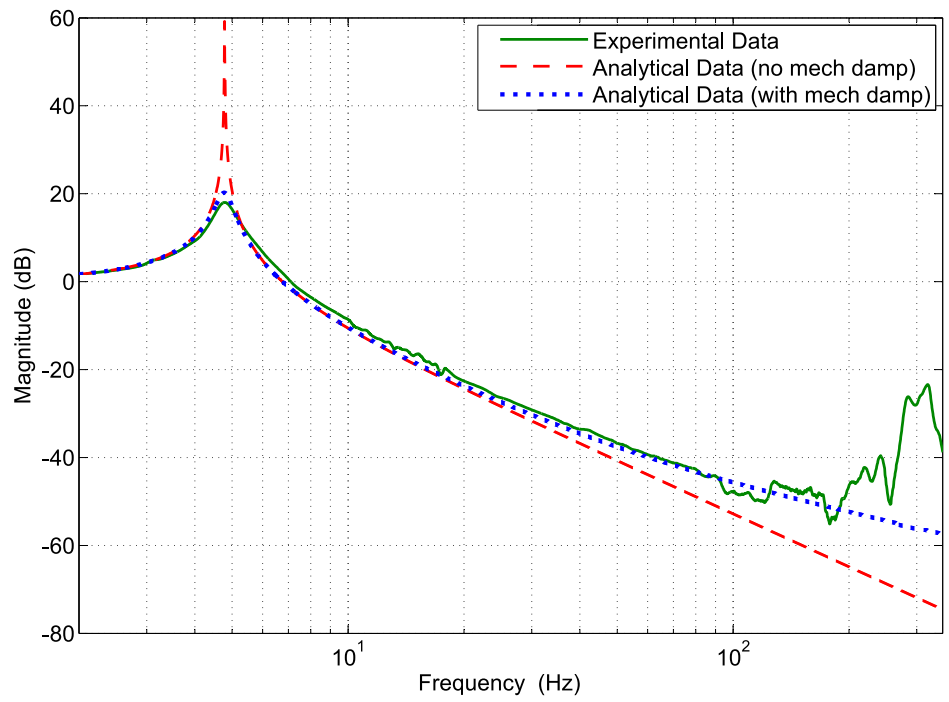

(a)

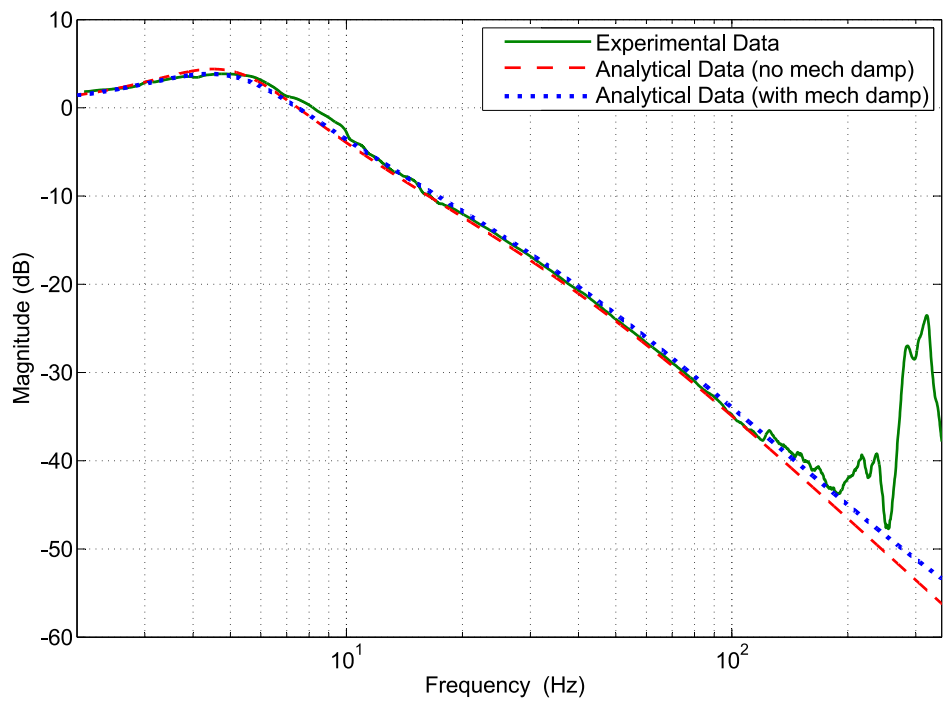

(b)

Figure 8: Comparison between the test results and the analytical model. The dashed line does not include the extra mechanical damping, whereas the dotted line does include it. (a) system without EMSD; (b) system with EMSD. 


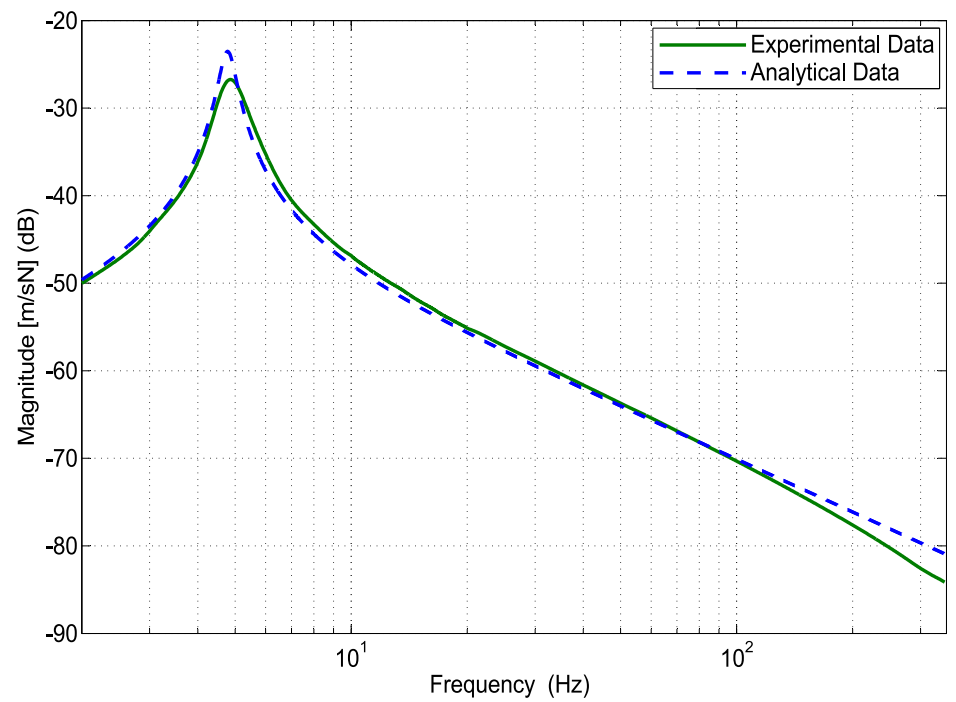

Figure 9: Transfer function between the input force and the mass velocity. The system considered is without EMSD.

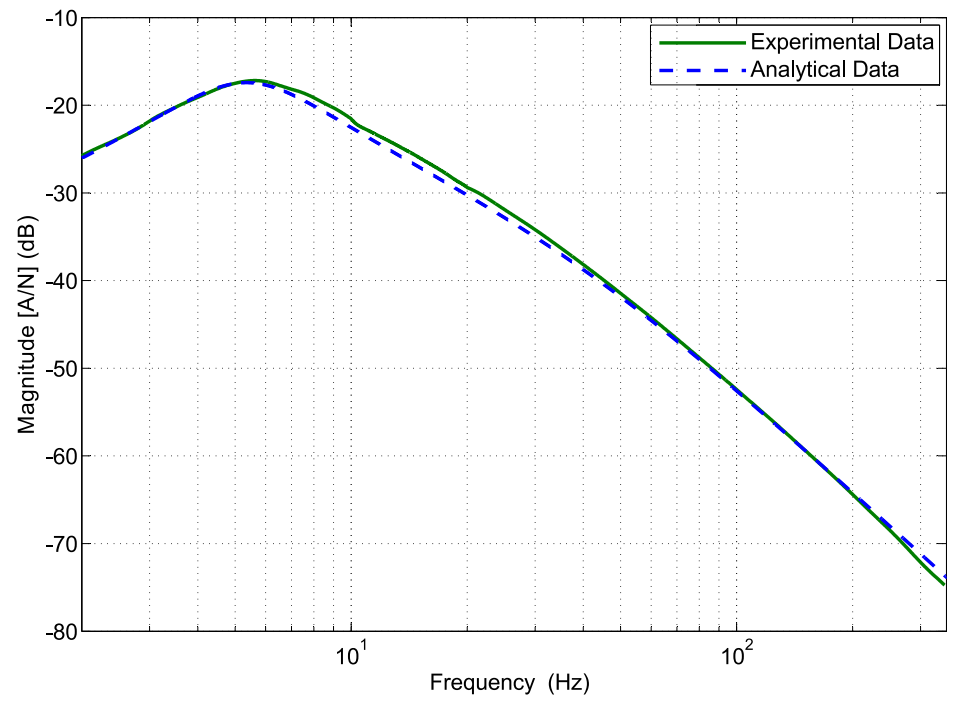

Figure 10: Transfer function between the input force and the induced current in the coil. The system considered is with EMSD. 


\section{Conclusions}

In the present work, a negative-resistance EMSD has been studied both analytically and experimentally. The proposed isolator uses a negative-resistance analogue circuit to reduce the inherent resistance of the electromagnetic coils, and as a result, the damping of the system can be increased. A 5-kg, SDOF system has been employed to demonstrate the performance of the proposed damper. The governing system of equations for this system has been established, and the relative state-space model has been reported. The electro-mechanical transducer coefficient has been analysed through the software COMSOL Multiphysics and its value has been increased by changing the disposition of magnets and iron yokes. A parametric trade-off has been carried out in order to meet the requirements of maximum amplification at the resonance peak and low cutoff frequency within the operating temperature range of $-20^{\circ} \mathrm{C}$ to $+50^{\circ} \mathrm{C}$. The test rig has been built in-house, and the results from a test campaign have corroborated the accuracy of the analytical model. This paper has demonstrated that this technology is capable of isolating micro-vibration sources (e.g. reaction wheel assemblies) without requiring an active control algorithm. The low required power, the simplified electronics and the small device mass make this EMSD extremely interesting for future space applications.

\section{Acknowledgements}

The authors would like to thank Dr. Marcello Remedia for his valuable and constructive suggestions during the different phases of this research work

\section{References}

[1] G. Richardson, G. Smet, and G. Aglietti, "Managing micro-vibration on the sstl300-s1 a $400 \mathrm{~kg} 1 \mathrm{~m}$ resolution earth imaging spacecraft," Proceeding of the 13th European Conference on Spacecraft Structures, Materials and Environmental Testing (SSMET), April 2014. 
[2] J. R. Maly, S. C. Pendleton, J. Salmanoff, G. J. Blount, and K. Mathews, "Hubble space telescope solar array damper," Proc. SPIE, vol. 3672, pp. 186-197, 1999.

[3] R. Bronowicki, A.J. andMacDonald, Y. Gursel, R. Goullioud, T. Neville, and D. Platus, "Dual stage passive vibration isolation for optical interferometer missionsn," Proceedings SPIE Interferometry in space, vol. 4852, pp. 753-763, Feb. 2003.

[4] J.-S. Bae, J.-H. Hwang, J.-S. Park, and D.-G. Kwag, "Modeling and experiments on eddy current damping caused by a permanent magnet in a conductive tube," Journal of Mechanical Science and Technology, vol. 23, no. 11, pp. 3024-3035, 2009.

[5] B. Ebrahimi, M. Khamesee, and F. Golnaraghi, "Permanent magnet configuration in design of an eddy current damper," Microsystem Technologies, vol. 16, no. 1-2, pp. 19-24, 2010.

[6] J.-S. Bae, M. K. Kwak, and D. J. Inman, "Vibration suppression of a cantilever beam using eddy current damper," Journal of Sound and Vibration, vol. 284 , no. 35 , pp. $805-824,2005$.

[7] P. Davis, D. Carter, and T. Hyde, "Second generation hybrid d-strut," Proceeding of SPIE Smart Structures and Material conference, 1995.

[8] G. Wilson and P. Wolke, "Performance prediction of d-strut isolation systems," Proceeding of SPIE Smart Structures and Material conference, vol. 3045, 1997.

[9] N. Hagood and A. von Flotow, "Damping of structural vibrations with piezoelectric materials and passive electrical networks," Journal of Sound and Vibration, vol. 146, no. 2, pp. 243 - 268, 1991.

[10] S.-y. Wu, "Piezoelectric shunts with a parallel r-l circuit for structural damping and vibration control," vol. 2720, pp. 259-269, 1996. 
[11] C. Liu, X. Jing, S. Daley, and F. Li, "Recent advances in micro-vibration isolation," Mechanical Systems and Signal Processing, vol. 5657, no. 0, pp. $55-80,2015$.

[12] G. S. Aglietti, S. B. Gabriel, R. S. Langley, and E. Rogers, "A modeling technique for active control design studies with application to spacecraft microvibrations," The Journal of the Acoustical Society of America, vol. 102, no. 4, 1997.

[13] G. Aglietti, P. Cunningham, and R. Langley, "Verification of various modelling techniques for simply-supported piezoelectric actuated thin panels," Proceedings of the Institution of Mechanical Engineers, Part G: Journal of Aerospace Engineering, vol. 216, no. 3, pp. 155-164, 2002.

[14] E. Anderson, J. Fumo, and R. Erwin, "Satellite ultraquiet isolation technology experiment (suite)," in Aerospace Conference Proceedings, 2000 IEEE, vol. 4, pp. 299-313 vol.4, 2000.

[15] S. M. Yang and C. A. Jeng, "Structural vibration suppression by concurrent piezoelectric sensor and actuator," Smart Materials and Structures, vol. 5, no. 6 , p. 806, 1996.

[16] A. Fleming and S. Moheimani, "Control oriented synthesis of electromagnetic shunt impedances for vibration isolation," IEEE transactions on Control Systems Technology, vol. 13, no. 1, 2005.

[17] J. J. Dosch, D. J. Inman, and E. Garcia, "A self-sensing piezoelectric actuator for collocated control,", Journal of intelligent materials and structures, vol. 3, no. 1, pp. 166 - 185, 1992.

[18] G. Hauge and M. Campbell, "Sensors and control of a space-based sixaxis vibration isolation system," Journal of Sound and Vibration, vol. 269, no. 35, pp. 913 - 931, 2004. 
[19] J. Spanos, Z. Rahman, and G. Blackwood, "A soft 6-axis active vibration isolator," in American Control Conference, Proceedings of the 1995, vol. 1, pp. 412-416 vol.1, Jun 1995.

[20] A. J. Fleming, S. O. R. Moheimani, and S. Behrens, "Synthesis and implementation of sensor-less active shunt controllers for electromagnetically actuated systems," Control Systems Technology, IEEE Transactions on, vol. 13, pp. 246-261, March 2005.

[21] S. Behrens, A. Fleming, and S. Moheimani, "Control orientated synthesis of electromagnetic shunt impedances for vibration isolation," IEEE/ASME Transactions on Mechatronics, vol. 9, no. 1, 2004.

[22] M. Neubauer, R. Oleskiewicz, K. Popp, and T. Krzyzynski, "Optimization of damping and absorbing performance of shunted piezo elements utilizing negative capacitance," Journal of Sound and Vibration, vol. 298, no. 12, pp. $84-107,2006$.

[23] S. Manzoni, S. Moschini, M. Redaelli, and M. Vanali, "Vibration attenuation by means of piezoelectric transducer shunted to synthetic negative capacitance," Journal of Sound and Vibration, vol. 331, no. 21, pp. 4644 4657, 2012.

[24] X. Zhang, H. Niu, and B. Yan, "A novel multimode negative inductance negative resistance shunted electromagnetic damping and its application on a cantilever plate," Journal of Sound and Vibration, vol. 331, no. 10, pp. $2257-2271,2012$.

[25] B. Yan, X. Zhang, Y. Luo, Z. Zhang, S. Xie, and Y. Zhang, "Negative impedance shunted electromagnetic absorber for broadband absorbing: experimental investigation," Smart Materials and Structures, vol. 23, no. 12, p. 125044, 2014.

[26] B. Yan, X. Zhang, and H. Niu, "Design and test of a novel isolator with 
negative resistance electromagnetic shunt damping," Smart Materials and Structures, vol. 21, no. 3, p. 035003, 2012.

[27] B. Yan, X. Zhang, and Y. Luo, "Investigation of negative resistance shunt damping for the vibration control of a plate," International Journal of applied electromagnetics and mechanics, vol. 45, no. 1, pp. 93-100, 2014.

[28] A. Preumont, M. Horodinca, I. Romanescu, B. de Marneffe, M. Avraam, A. Deraemaeker, F. Bossens, and A. A. Hanieh, "A six-axis single-stage active vibration isolator based on stewart platform," Journal of Sound and Vibration, vol. 300, no. 35, pp. $644-661,2007$.

[29] H. Sodano, J.-S. Bae, D. Inman, and W. Belvin, "Concept and model of eddy current damper for vibration suppression of a beam," Journal of Sound and Vibration, vol. 288, no. 45, pp. 1177 - 1196, 2005.

[30] P. Horowitz and W. Hill, the art of electronics. New York: Cambridge University Press, 1996.

[31] J.-S. Bae, J.-H. Hwang, J.-H. Roh, and M.-S. Yi, "Development of an electromagnetic shock absorber," International Journal of applied electromagnetics and mechanics, vol. 49, pp. 157-167, 2015. 